\title{
A Comparitive Study of Alvarado Score And Ripasa Score in the Diagnosis Of Acute Appendicitis
}

\author{
R.S RAIKWAR ${ }^{1}$, VARSHA DHAKAD $^{2}$, SANJAY SINGH $^{[3}$, R.K MATHUR $^{4}$ \\ ${ }^{1}$ Associate Prof. Department Of Surgery, MGM Medical College And M.Y. Hospital, Indore \\ ${ }^{2}$ Asst. Prof. Department Of Surgery, MGM Medical College And M.Y. Hospital, Indore \\ ${ }^{3}$ Resident, Department Of Surgery, MGM Medical College And M.Y. Hospital, Indore \\ ${ }^{4}$ Professor And HOD, Department Of Surgery, MGM Medical College And M.Y. Hospital, Indore
}

\begin{abstract}
Acute Appendicitis is one of the most common surgical emergencies in clinical practice with estimated life time prevalence approximately 1 in $7 .{ }^{[1]}$ The incidence is 1.5 to 1.9 per 1000 and is approximately 1.4 times greater in men then in women. ${ }^{[2]}$

Aim: This study was undertaken to compare Alvarado and RIPASA score to diagnose acute appendicitis ,to know the efficacy of individual score for early diagnosis of acute appendicitis. to improve the diagnostic criteria for acute appendicitis

Material and Methods: The comparative study of two scores in the diagnosis of acute appendicitis involved 200 patients admitted in teaching hospital.

Results: RIPASA scoring system is more sensitive (98.42\%) as compared to the Alvarado scoring system (73.7\%). RIPASA score is more specific (90\%) as compared to Alvarado scoring system (80\%). PPV of RIPASA scoring system is $99.46 \%$ as compared to $94.32 \%$ of the Alvarado scoring system. NPV of the RIPASA scoring system is $75 \%$ while that of Alvarado scoring system is 3.38\%. ROC analysis depicts the cut off point for the diagnosis with maximum sensitivity and specificity for the RIPASA scoring system to be at 7 which is different from the original cut off value of 7.5 .

Conclusion: Both Alvarado and RIPASA score have significant difference when Non-Parameteric Pearson Chi Square TEST was applied with $p$ value $<0.05$, fairly the correlation between the Alvarado and RIPASA Score rho $=0.538$ and $p$ value $<0.05$, significant difference was found with positive agreement between the two scores .From the above parameters we conclude that in our present study RIPASA Score is a better Score in diagnosing the cases of acute appendicitis as compared to the Alvarado score. Still due to lack of studies done for RIPASA Score we need more studies to be conducted on Asian population to prove that the new score is a better score in diagnosing acute appendicitis .
\end{abstract}

\section{Introduction}

Acute Appendicitis is one of the most common surgical emergencies in clinical practice with estimated life time prevalence approximately 1 in $7 .{ }^{[1]}$ The incidence is 1.5 to 1.9 per 1000 and is approximately 1.4 times greater in men then in women. ${ }^{[2]}$ Despite being a common problem, acute appendicitis remains a difficult diagnosis to establish, particularly in young, elderly and in the females of reproductive age group where genitourinary and gynaecological conditions can present with similar signs and symptoms as those of acute appendicitis. History and clinical examination both remain the most effective and practical diagnostic modalities. ${ }^{[3]}$ Acute appendicitis is associated with raised TLC. It is raised in other inflammatory conditions also, making its role only supportive in the diagnosis of acute appendicitis. ${ }^{[4]}$ There has been a need of a scoring system that can overcome these problems, with good sensitivity and specificity and acceptable negative appendicectomies on exploration. One of the common scoring system is Alvarado system which is based on clinical and laboratory investigations of acute appendicitis includes pain migration to RIF, anorexia, nausea and vomiting, tenderness, rebound tenderness, fever and leukocytosis and shift of WBC to the left. ${ }^{[5]}$ Another scoring system RIPASA score has been derived for Asian countries that takes into consideration for age, sex, urine analysis, guarding, Rovsing sign, Asian origin in addition to the variables in Alvarado score. ${ }^{[6]}$

\section{Patients And Methods}

The present study consists of 200 patients of age group 15 to 60 years diagnosed with acute appendicitis between April 2015 and March 2016 in M.Y Hospital ,INDORE,(M.P).

\section{Methods}

The Study was conducted in Department of Surgery in M.G.M Medical College and M.Y Hospital, Indore from April 2015 to March 2016. Ethical committee clearance was taken for the study.This is a prospective study of all patients coming to our institution in the Department of Surgery with complaints of right 
iliac fossa abdominal pain. The basic aim is to compare the Alvarado Score and RIPASA Score in their efficacy to diagnose acute appendicitis so that the diagnostic criteria can be improved and we can reduce the unwanted operations and expensive imaging studies .

\begin{tabular}{|l|l|}
\multicolumn{2}{l}{ Alvarado Score } \\
\hline Characteristics & Score \\
\hline $\begin{array}{l}\text { M - Migration Of Pain To The Right Lower } \\
\text { Quadrant }\end{array}$ & 1 \\
\hline A - Anorexia & 1 \\
\hline N - Nausea & 1 \\
\hline T - Tenderness In The Right Lower Quadrant & 2 \\
\hline R - Rebound Tenderness & 1 \\
\hline E - Elevated Temperature & 1 \\
\hline L - Leucocytosis & 2 \\
\hline S - Shift Of Wbc To The Left & 1 \\
\hline Total & $\mathbf{1 0}$ \\
\hline
\end{tabular}

Interpretation of Alvarado score

\begin{tabular}{|l|l|}
\hline Score & Interpretation \\
\hline $1-4$ & Very unlikely, keep under observation \\
\hline $5-6$ & Acute appendicitis, may be, for regular observation \\
\hline $7-8$ & Acute appendicitis is probable, operate \\
\hline $9-10$ & Acute appendicitis definite, operate \\
\hline
\end{tabular}

\begin{tabular}{|l|l|}
\hline \multicolumn{2}{|c|}{ RIPASA Score } \\
\hline Parameter & Score \\
\hline SEX & \\
Male & 1.0 \\
Female & 0.5 \\
\hline AGE & \\
$<39.9 \mathrm{yrs}$ & 1.0 \\
$>$ 40.0yrs & 0.5 \\
\hline RIF PAIN & 0.5 \\
\hline MIGRATION OF RLQ PAIN & 0.5 \\
\hline ANOREXIA & 1.0 \\
\hline NAUSEA AND VOMITING & 1.0 \\
\hline DURATION OF SYMPTOMS & \\
<48hrs & 1.0 \\
\hline$>48$ hrs & 0.5 \\
\hline RIF TENDERNESS & 1.0 \\
\hline RIF GUARDING & 2.0 \\
\hline REBOUND TENDERNESS & 1.0 \\
\hline ROVSING S SIGN & 2.0 \\
\hline FEVER & 1.0 \\
\hline RAISED WBC & 1.0 \\
\hline NEGATIVE URINALYSIS & 1.0 \\
\hline FOREIGN NRIC & 1.0 \\
\hline
\end{tabular}

Minimal total score is 2, Maximum total score is 17.5 . The receiver operating curve (ROC) at the optimal cutoff threshold score for the new appendicitis scoring system was derived and 7.5 was the cut-off.

Age Group

Table No. 1

\begin{tabular}{|l|l|}
\hline AGE Groups & No. of Patients in particular AGE Group \\
\hline $15-25$ & $90(45 \%)$ \\
\hline $26-35$ & $53(26.5 \%)$ \\
\hline $36-45$ & $37(18.5 \%)$ \\
\hline $46-60$ & $20(10 \%)$ \\
\hline
\end{tabular}


Out of 200 patients 90 patients are between age group 15-25 years, 53 between 26-35years,37 between 36-45years, 20 between 46-60 years .

Male : Female Ratio

Table No. 2

\begin{tabular}{|l|l|l|}
\hline & M A L E S & F E M A L E S \\
\hline NUMBER & 132 & 68 \\
\hline PERCENTAGE & $66 \%$ & $34 \%$ \\
\hline
\end{tabular}

Out of 200 patients 132 are Males and 68 are Females.

\section{Signs and Symptoms}

Table no. 3

\begin{tabular}{|l|l|}
\hline Signs and Symptoms & No. of Patients \\
\hline Pain in Abdomen & 195 \\
\hline Nausea and Vomiting & 176 \\
\hline Anorexia & 156 \\
\hline Fever & 132 \\
\hline RIF Tenderness & 172 \\
\hline Rebound Tenderness & 60 \\
\hline Gaurding & 68 \\
\hline Rovsing Sign & 64 \\
\hline
\end{tabular}

Pain in abdomen is present in 195 (97.5\%) patients, Nausea and Vomiting in 176 (88\%),Anorexia in 156 (78\%),Fever in 132 (66\%),RIF Tenderness in 172 (86\%), Rebound Tenderness in 60 (30\%), Guarding in 68 (34\%), Rovsing's Sign in 64 (32\%) patients .

Post OperativeHistopathalogy of Appendix

\begin{tabular}{|l|l|}
\hline Histopathalogy & No. of Patients \\
\hline Positive (+ve) & 190 \\
\hline Negative (-ve) & 10 \\
\hline
\end{tabular}

Out of 200 patients 190 patients tested positive for Acute Appendicitis and 10 patients had normal appendix

Treatment Modality

\begin{tabular}{|l|l|}
\hline Treatment Modality & No. of Patients \\
\hline Emergency Appendicectomy & 193 \\
\hline Exploratory Laparotomy & 7 \\
\hline
\end{tabular}

\section{Results}

The study period was 12 months with total 200 patients were involved. Total 200 patients of age range 15 to 60 years of age. Peak age group was 15 to 25 years of age (45\%). Least affected age group was above 45 years of age. Males are predominantly affected with M:F ratio 1.9:1.Most common presentation was found Pain in abdomen in $97.5 \%$ of patients followed by Nausea and Vomiting in $88 \%$ and Right Iliac Fossa Tenderness in $86 \%$ of the patients .Majority of patients $(58 \%)$ presenting after 48 hours of the onset of symptoms to the hospital.

WBC counts were found to be raised (>10,000/mm3)in $56 \%$ of the patients .In $94 \%$ of the patients Urine culture and sensitivity was found positive.Almost $90 \%$ of the patients were diagnosed positive for acute appendicitis on Ultrasonography. In Histopathology, 95\% of the patients tested positive for acute appendicitis. Emergency Appendicectomy were performed in about 193 (96.5\%) patients and 190 (95\%)patients confirmed Histology for acute appendicitis. Mean Hospital stay was 3.05 days. Wound infection was found in $2.5 \%$ of the patients, $2 \%$ having Wound Gaping and $2 \%$ had Postoperative adhesions. Most of the patients $(65 \%)$ were discharged within 4 days of admission. Regarding Alvarado Score and RipasaScore,Out of 200 patients $108(54 \%)$ patients had Alvarado Score $<7$ and $92(46 \%)$ had score $>=7$ and 3(1.5\%) patients had RIPASA Score $<5,12(6 \%)$ patients have scores between 5-7, 133(66.5\%) patients had scores between 7.5-11.5 , 55(27.6\%) patients had scores $>=12$. 


\section{Discussion}

The present study included clinically suspected 200 cases of appendicitis, with age group of patients taken from 15 to 60 years of age. There were 132 males and 68 females in the study. All the patients clinically suspected to have acute appendicitis were scored according to both the scoring systems and were taken up for surgery. Histopathology was considered the gold standard for the confirmation of the diagnosis. The histopathologically inflamed appendix was classified acute appendicitis. The histopathologically normal appendix was put under no appendicitis group.

The symptoms such as RIF pain was present in 195 patients (97.5\%) in the study group. Pain migration was present in 37 (74\%), anorexia was present in 156 patients (78\%), nausea and vomiting was present in 146 patients (73\%), fever was present in 132 patients (66\%) and presenting with duration of symptoms less than 48 hours were $84(42 \%)$. Of all the symptoms RIF pain, Pain migration, nausea and vomiting and duration of symptoms came out to be statistically significant (with $\mathrm{p}$ value $0.032,0.048,0.016$ and 0.042 respectively) but RIF pain and nausea and vomiting came out to be highly significant (with p value 0.032 and 0.016 respectively) Signs such as RIF tenderness was present in 176 cases ( $p$ value 0.016 ), guarding was present in 68 (p value 0.226 ), rebound tenderness was present in 61 ( $\mathrm{p}$ value 0.461 ) and Rovsing `s sign was present in 64 (p value 0.134). Out of all the clinical signs, RIF tenderness was found to be statistically significant.

Alvarado score when applied in all patients clinically suspected to have appendicitis, had 92 cases (46\%) with a score of $\geq 7$ and 108 cases (54\%) with a score of <7. On analyzing with respect to the histopathology, the sensitivity and specificity of the scoring system in the present study came out to be $70 \%$ and $20 \%$ respectively. The positive and negative predictive values were $94.32 \%$ and $3.38 \%$ respectively. Accuracy was $74 \%$ with 8 false positive cases and 57 false negative cases. Khan et al applied the Alvarado scoring system in Asian population and achieved a sensitivity and specificity of 59\% and $23 \%$ respectively, with a positive predictive value of $83.3 \%$ with negative appendicectomy rate of $15.6 \% .[59]$

RIPASA score when applied in all the patients clinically suspected of having appendicitis, had 188 patients $(94 \%)$ in $\geq 7.5$ group and 12 patients $(6 \%)$ in $<7.5$ score group. When analyzed with respect to histopathology the sensitivity of the scoring system in the present study came to be $98.42 \%$, specificity of $90 \%$, positive and negative predictive values of $99.46 \%$ and $75 \%$ respectively. In the retrospective study by Chong CF et al ROC analysis quoted that the expected sensitivity and specificity of the RIPASA scoring system were $88 \%$ and $67 \%$ respectively with a diagnostic accuracy of $81 \%$. The positive and negative predictive values were expected to be $93 \%$ and $53 \%$ respectively. ${ }^{[6]}$

The findings of the present study match with the Chong CF et al study. On comparing both the scoring systems in the present study, RIPASA score has been found to be more sensitive (98.42\%) as compared to Alvarado score (73.7\%). RIPASA score is also more specific (90\%) as compared to Alvarado score (80\%). Positive and negative predictive values of RIPASA came out to $99.46 \%$ and $75 \%$ as compared to Alvarado having $94.32 \%$ and $3.38 \%$. Accuracy of the RIPASA score was $97 \%$ as compared to the Alvarado score having accuracy of $74 \%$.In a prospective study by Chon CF et al, the sensitivity, specificity, positive predictive value, negative predictive value and diagnostic accuracy of the RIPASA score were 98\%, 81.3\%, 85.3\%, 97.4\% and 91.8\% respectively when compared to Alvarado score with sensitivity, specificity, positive predictive value, negative predictive value and diagnostic accuracy of $68.3 \%, 87.9 \%, 86.3 \%, 71.4 \%$ and $86.5 \%$ respectively. The authors of the RIPASA scoring system have claimed in this comparative prospective study that RIPASA score is better than Alvarado score in Asian settings. ${ }^{[7]}$ ROC analysis was done in the present study to look for the cut off scores for both the scoring systems, with good sensitivity and specificity.Alvarado score cut off was 6.5 for maximum sensitivity and specificity, which was lower than the original cut off value off $7 \cdot{ }^{[5]}$ In a study by Jang $\mathrm{SO}$ et al cut off for maximum sensitivity and specificity was 6 which was lower than the original cut off value of 7 , suggesting a mild ethnic difference with regard to Alvarado score.

RIPASA score cut off value came out to be 7 , which is a little lower than the original cut off value of $7.5{ }^{[6]}$ The sensitivity and specificity at the cut off value of 7 came out to be $98.42 \%$ and $90 \%$ respectively, when compared to $95.6 \%$ and $80 \%$ respectively at the cut off value of 7.5 in the present study.

\section{Conclusion}

From the above parameters we conclude that in our present study RIPASA Score is a better Score in diagnosing the cases of acute appendicitis as compared to the Alvarado score. Still due to lack of studies done for RIPASA Score we need more studies to be conducted on Asian population to prove that the new score is a better score in diagnosing acute appendicitis.

\section{References}

[1]. Stephens PL and Mazzuco JJ. Comparison of ultrasound and the Alvarado score for the diagnosis of acute appendicitis. Conn Med 1999;63:137-40

[2]. Cuschieri A. The small intestine and vermiform appendix. In: Cuschieri A, G R Giles, A R s 
[3]. Peterson MC, Holbrook JH, Hales D, Smith NL, Staker LV. Contributions of history, physical examination and laboratory investigations in making medical diagnosis. West J Med. 1992;156(2):163-5.

[4]. Lau WY, Ho YC, Chu KW, Yeung C. Leucocyte count and neutrophil percentage in appendicectomy for suspected appendicitis. Aust N Z J Surg. 1989;59(5):395-8.

[5]. Alvarado A. A practical score for early diagnosis of acute appendicitis. Ann Emerg Med. 1986;15:557-64.

[6]. Chong CF, Adi MIW, Thien A, Suyoi A, Mackie AJ, Tin A S et al. Development of the RIPASA score: a new appendicitis scoring system for the diagnosis of acute appendicitis. Singapore Med J. 2010;51:220-5

[7]. Chong CF, Thien A, mackie AJ, Tin AS, Tripathi S, Ahmad MA, Tan LT, Ang SH, Telisinghe PU. Comparison of RIPASA and Alvarado scores for the diagnosis of acute appendicitis. Singapore Med J. 2011;52(5):340-5. 